\title{
Testosterone, Cortisol and Empathy: Evidence for the Dual-Hormone Hypothesis
}

\author{
Samuele Zilioli • Davide Ponzi • Andrea Henry • \\ Dario Maestripieri
}

Received: 27 September 2014 / Revised: 3 November 2014 / Accepted: 10 November 2014 /

Published online: 19 November 2014

C) Springer International Publishing 2014

\begin{abstract}
The dual hormone hypothesis posits that basal cortisol and testosterone have a joint effect on motivational and behavioral systems implicated in dominance and aggression, such that traits generally associated with high testosterone manifest more in individuals with low basal cortisol levels. Whether this hypothesis applies to behavioral systems other than dominance remains unclear. In the present study, we investigated the association between basal testosterone, basal cortisol, and empathy in a large population of MBA students. Empathy was assessed with a short version of the Davis's Interpersonal Reactivity Index and with the Reading the Mind in the Eyes Test (RMET). Higher testosterone was predictive of lower empathy scores among men and women with low basal cortisol, while this association was reversed among individuals with high cortisol levels. In other words, a high-testosterone profile was found to be predictive of both high and low empathic dispositions depending on the concomitant HPA state. The effect was limited to self-reported empathy as no association was found with the RMET. This pattern of results, which emerged when data for men and women were analyzed together, remained significant only for men when analyses were run separately for the two sexes. These results add empathy to the list of behaviors regulated by the joint action of testosterone and cortisol, as outlined by the dual hormone hypothesis.
\end{abstract}

Keywords Testosterone $\cdot$ Cortisol $\cdot$ Empathy D Dual-hormone hypothesis

\section{Introduction}

In order to navigate successfully the complexity of our social environment it is crucial to attempt to understand other people's mental and emotional states and then act

\footnotetext{
S. Zilioli $(\bowtie)$

Department of Psychology, Wayne State University, 5057 Woodward Ave, 7th Floor, Detroit, MI 48202, USA

e-mail: sam.zilioli@gmail.com

D. Ponzi $\cdot$ A. Henry $\cdot$ D. Maestripieri

Institute for Mind and Biology, The University of Chicago, Chicago, IL, USA
} 
accordingly, for example, by approaching individuals in need of help or avoiding others with intentions to harm. The cognitive ability that helps us understand or guess the thoughts, intentions, or desires of another person is referred to as "cognitive empathy" (or theory of mind; Gonzalez-Liencres et al. 2013), while the ability to feel into others' states (i.e. emotional resonation or emotional resonance) is referred to as "emotional empathy" (Baron-Cohen 2009; Buchanan et al. 2012).

Comparative and evolutionary research on empathy, as well as research on the possible neural and physiological substrates of empathy, has suggested that empathy is biologically rooted in, and, at least partially regulated by, the endocrine system (Preston and De Waal 2002; Yildirim and Derksen 2012; Gonzalez-Liencres et al. 2013). Generally, women outperform men in tasks of emotional and cognitive empathy and tend to self-report higher dispositional empathy than men (Davis 1983; BaronCohen et al. 2001; Derntl et al. 2010). Men, on the other hand, are more likely than women to suffer from social cognition disorders such as Autism Spectrum Disorders (Baron-Cohen et al. 2005) and psychopathy (Cale and Lilienfeld 2002), which are characterized by deficits in empathy. Since men, on average, have much higher testosterone than women, it is possible that testosterone is inversely related to empathy and that sex differences in empathy are accounted for, at least in part, by sex differences in testosterone. In terms of mechanisms, testosterone can affect the activity of the amygdala, resulting in reduced emotional empathy (Terburg et al. 2009).

The hypothesized negative association between empathy and testosterone is at the basis of the "extreme male brain" theory of autism, which posits that the deficits in cognitive and emotional empathy that are characteristics of ASD result from the organizational effects of fetal testosterone (Baron-Cohen et al. 2005). Consistent with this hypothesis, studies have shown that in infants and children, fetal testosterone (measured through amniocentesis) was negatively correlated with amount of eye contact made with their parents (Lutchmaya et al. 2002) and with scores of the "Reading the Mind in the Eyes Test" (RMET) (Chapman et al. 2006). There is also evidence that activational effects of testosterone may play a role in cognitive empathy later in life. For example, treatment with exogenous testosterone has been shown to reduce empathic responses in women in a test that measures the automatic, unconscious mirroring of another's motor actions (Hermans et al. 2006; van Honk and Schutter 2007). Moreover, the effects of exogenous testosterone on empathy are stronger in women exposed to higher prenatal androgens (Van Honk et al. 2011), suggesting that there may be interactions between the organizational and the activational effects of testosterone on empathy.

In addition to testosterone, the hypothalamus-pituitary-adrenal (HPA) axis may also be involved in the neuroendocrine regulation of empathy. The HPA axis plays an important role in the stress response by releasing glucocorticoids (e.g., cortisol), which fuel the organism with the energy required to face a stressful situation (Sapolsky et al. 2000). Although few studies have specifically, mostly indirectly, investigated the effects of cortisol on empathy (Tennes and Kreye 1985; Stallings et al. 2001; Adam and Gunnar 2001; Nakayama et al. 2007; Booth et al. 2008; Barraza and Zak 2009; Buchanan et al. 2012), it has been suggested that the expression of emotional empathy may correlate with high basal cortisol concentrations as well as with stress-induced cortisol increase. For example, Buchanan and colleagues (Buchanan et al. 2012) reported that individuals observing a Trier Social Stress Test (TSST), a task involving 
a formal job interview and an arithmetic test aimed at evoking psychological stress and cortisol secretion, showed increases in cortisol similar to those of the subjects who were taking the test.

Testosterone and cortisol may influence empathy separately, or jointly. The activities of the HPA and the hypothalamus-pituitary gonadal (HPG) axes, which lead to the secretion of cortisol and testosterone respectively, are intrinsically co-regulated. Specifically, cortisol can inhibit the secretion of testosterone at all the levels of the HPG axis, while testosterone can act upon the hypothalamus to inhibit cortisol release (Viau 2002). An imbalance between cortisol and testosterone in the direction of high baseline levels of testosterone and low baseline levels of cortisol is correlated with features of psychopathy (Van Honk and Schutter 2006; Terburg et al. 2009). Based on these studies of testosterone and empathy, it could be hypothesized that high testosterone and low cortisol should be associated with lower empathy. This idea would also be congruent with the dual-hormone hypothesis proposed by Mehta and Josephs (Mehta and Josephs 2010), according to which physiological (Mehta and Josephs 2010; Zilioli and Watson 2012) and behavioral (Dabbs et al. 1991; Popma et al. 2007; Mehta and Josephs 2010) aspects of dominance should be more pronounced in individuals with high baseline testosterone and low baseline cortisol levels.

In the present study, we investigated the relationship between basal testosterone, basal cortisol, and empathy in a large population of MBA students at a private university. We used both a self-reported measure of empathy and an experimental one. We tested the hypothesis that men and women with higher testosterone and lower cortisol should score lower on empathy.

\section{Methods}

Five-hundred and one MBA students (348 males; mean age $=28.73, \mathrm{SD}=0.13 ; 153$ females; mean age $=27.42, \mathrm{SD}=0.20$ ) from the Booth Business School at the University of Chicago served as study participants. Data for the empathy measures were missing for 32 individuals, leaving a total of 469 participants (323 males, 146 females). All students gave informed written consent for their participation in the study and were paid \$20 or more for their participation. The use of human subjects was approved by the Social Science IRB of the University of Chicago.

\section{Procedure}

This study was part of a larger investigation in which students were asked to take a 90min computerized test in which they played games that assessed their economic decision-making tendencies in different domains (see, Sapienza et al. 2009). Students were randomly selected to participate in one of two separate afternoon sessions. Starting time for the first session was 1:30 PM and 3:30 PM for the second session, and all sessions used an identical protocol (i.e. same order of the test battery). In addition to the computerized test, students took the Reading the Mind in the Eyes Test (RMET; Baron-Cohen et al. 2001), which measures an individual's ability to attribute mental states to others by recognizing their emotion from the eye region of the face, and completed several questionnaires, including one about empathy: the Interpersonal 
Reactivity Index (IRI) (Davis 1983). The IRI is made up of four seven-item scales, each measuring a different aspect of empathy: perspective taking, empathic concern, personal distress, and fantasy. Similar to other studies (e.g., Fabes et al. 1993) a shortened version of this questionnaire was utilized. We selected only a few items from a larger empathy scale to reduce the time and effort by study participants to complete the large number of tests and questionnaires included in this project. The choice of the four items, each of which was answered on a one to five Likert scale, was made on the basis of a pilot study we conducted prior to data collection for this project. Two items of the Empathic Concern Scale (item 1: "I often have tender, concerned feelings for people less fortunate than me"; item 2: "sometimes I don't feel very sorry for other people when they are having problems"; ordinal $\alpha=0.49$ ) and two items from the Perspective Taking Scale (item 1: "I sometimes try to understand my friends better by imagining how things look from their perspective"; item 2: "when I'm upset at someone, I usually try to "put myself in his shoes" for a while"; ordinal $\alpha=0.79)$ were chosen. The scores on the two empathic concern items and the two perspective taking items were added together (ordinal $\alpha=0.64$ ) for the purposes of data analyses (for a similar procedure, see Jackson et al. 2005).

\section{Saliva Collection and Hormones Analysis}

A saliva sample for baseline hormone measures was collected at the beginning of the test by passive drool. Plastic vials were stored frozen at $-80{ }^{\circ} \mathrm{C}$ until assayed in Dr. Chatterton's Endocrinology Laboratory at Northwestern University. On the day of the assay, samples were thawed and centrifuged. Salivary concentration of testosterone and cortisol were assayed by radioimmunoassay (RIA) using antisera prepared within the lab. Cross-reactivity for cortisol with corticosterone was null. Cross-reactivity for testosterone and other androgens was minimal. Sensitivity of the assays was $0.07 \mathrm{ng} /$ $\mathrm{mL}$ for cortisol and $7.5 \mathrm{pg} / \mathrm{mL}$ for testosterone. Intra-assay coefficients of variation were $\leq 10 \%$ for both hormones. Similarly, inter-assay coefficients of variation were $\leq 15 \%$. All samples were assayed in duplicate, and the average of duplicates was used in all analyses.

\section{Statistical Analysis}

Cortisol data were skewed for both men and women, while testosterone data were skewed only in women. For this reason, analyses were run separately in men, for which only cortisol was log-transformed, and women, for which both cortisol and testosterone were log-transformed. Individuals with baseline testosterone, cortisol, or empathy measures above or below three SDs from the mean for their gender were excluded as outliers. A total of 12 outliers ( 8 men, 4 women) were excluded, leaving a sample of 457 participants. ${ }^{1}$ T-tests were used to test group differences in hormonal and empathic measures. Multiple regressions were run to test the extent to which sex, testosterone, cortisol, and their interactions influence the measures of empathy. To interpret significant interactions we used tests for simple slopes (Aiken and West 1991; Meier 2008).

\footnotetext{
${ }^{1}$ When testing hypotheses on the entire sample raw testosterone data were used, leading the total number of female outliers to 8 (final $n=453$ ).
} 
The threshold for statistical significance in all analyses was set at a $p$ value of .05 (twotailed, in the case of t-tests).

\section{Results}

There were significant sex differences in hormone concentrations and trait empathy (shortened IRI). Men showed both higher testosterone [M women=42.88, $\mathrm{SD}=20.39$; $\mathrm{M}$ men=103.08, SD: 38.49; $\mathrm{t}(435.549)=-21.670, p<0.001, d=-1.95]$ and higher cortisol levels than women $[\mathrm{M}$ women=1.76, $\mathrm{SD}=.429$; $\mathrm{M}$ men=2, SD: .48; $\mathrm{t}(451)=-5.134$, $p<0.001, d=-0.52]$. In contrast, women scored higher than men on trait empathy [M women=14.72, $\mathrm{SD}=2.59 ; \mathrm{M}$ men=13.88, $\mathrm{SD}: 2.68 ; \mathrm{t}(451)=3.131, p=0.002, d=0.32]$. Men and women did not differ significantly in their RMET scores [M women=27.07, $\mathrm{SD}=3.45 ; \mathrm{M}$ men=26.7, $\mathrm{SD}=3.41 ; \mathrm{t}(451)=1.032, p=.303, d=0.11]$. Correlations among hormonal and empathic measures are reported in Table 1.

Hierarchical linear regressions were used in which empathy and RMET were the dependent variables and testosterone, cortisol, and their interaction were the potential predictors. In our first model, trait empathy was regressed on cortisol (standardized) and testosterone (standardized), which were entered as predictors in Step 1, while their interaction was entered in Step 2. In men, this analysis indicated that only the second linear regression model was significant as adding the interaction $[\beta=.135, p=.017]$ increased the amount of variance explained $\left[\left(\Delta \mathrm{F}(1311)=5.774, p=0.017, \Delta \mathrm{R}^{2}=\right.\right.$ $1.8 \%]{ }^{2}$ The same results were obtained when outliers were included $[(\Delta \mathrm{F}$ $(1319)=4.466, p=0.035, \Delta \mathrm{R}^{2}=1.4 \%$. In women, none of the models was significant and, consequently, no cortisol by testosterone interaction was found in Step 2 [ $\beta=-.008, p=.924]$. The same hierarchical regression model was run to predict RMET; however, none of the models was significant and, consequently, no cortisol by testosterone interaction was found in Step 2 for either men $[\beta=.000, p=.996]$ or women $[\beta=.054, p=.536]$.

To interpret the significant interaction between testosterone and cortisol in predicting men's IRI, we first computed simple slopes between empathy and cortisol 1 SD below and $1 \mathrm{SD}$ above the testosterone mean. As expected, for baseline testosterone below the mean, the slope did not significantly differ from zero $[\beta=-.033, p=.683]$, while a significant effect was found for baseline testosterone one SD above the mean $[\beta=.242$, $p=.003$ ], reflecting a significant positive association between basal cortisol and empathy at high concentrations of basal testosterone (Fig. 1). Simple slope analyses between empathy and testosterone one SD below and one SD above the cortisol mean revealed that the slope associated with high cortisol (i.e. 1 SD above the mean) -but not the slope associated with low cortisol $[\beta=-118, p=.175]$ - was statistically different from zero $[\beta=.157, p=.04]$.

To address the possibility that the joint effect of testosterone and cortisol on trait empathy was not observed in women because of the relatively smaller sample size we

\footnotetext{
${ }^{2}$ Interestingly, when running multiple regressions separately for the empathic concern scale and the perspective taking scale, a significant interaction emerged for the former $[\beta=.145, p=.010]$, but not the latter $[\beta=.071$, $p=.213]$. This suggests that the results on trait empathy were mainly driven by the conjoint effect of testosterone and cortisol on empathic concern.
} 


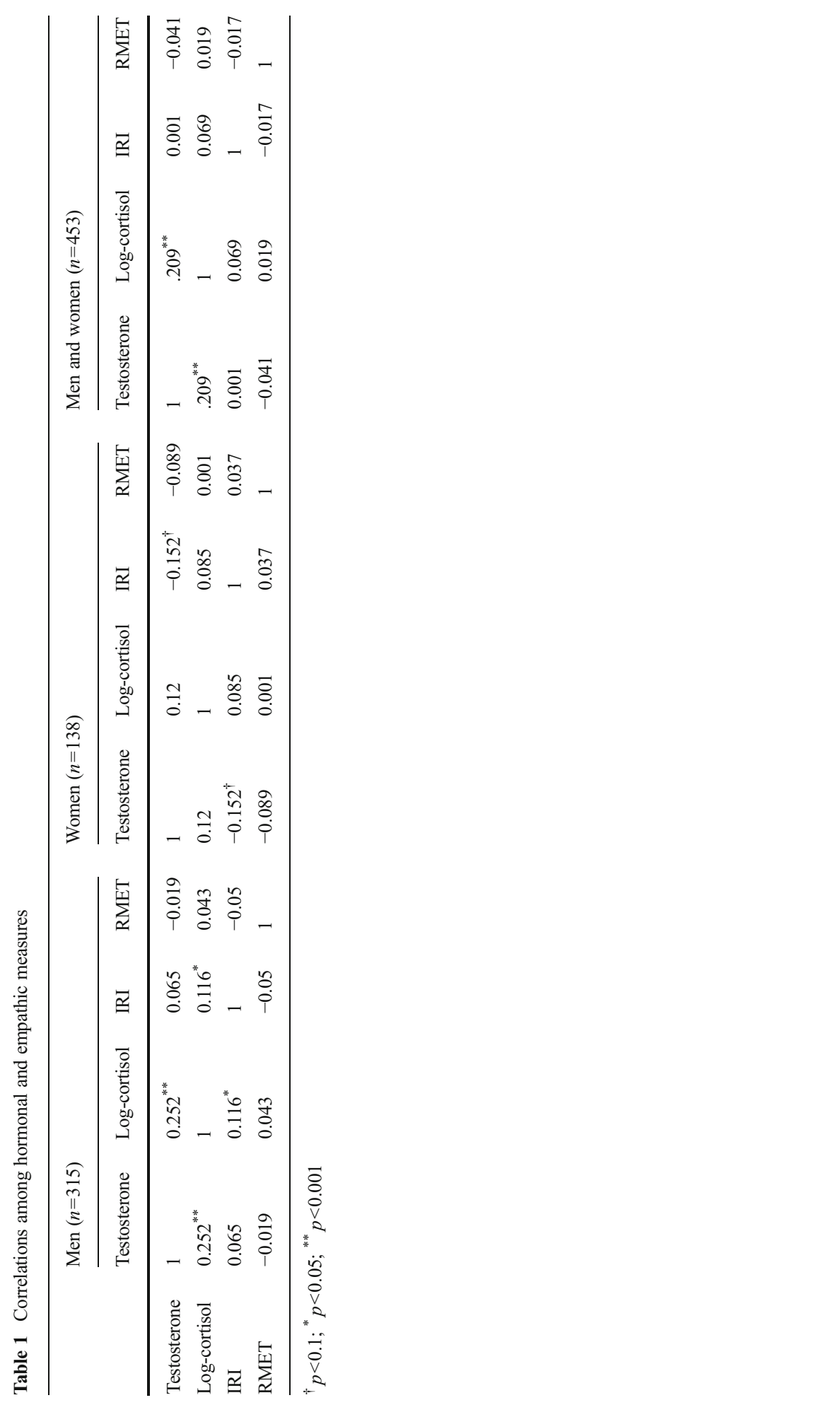


Fig. 1 Trait empathy as a function of basal testosterone and cortisol levels in men. When basal testosterone was high, basal cortisol was positively related to empathy

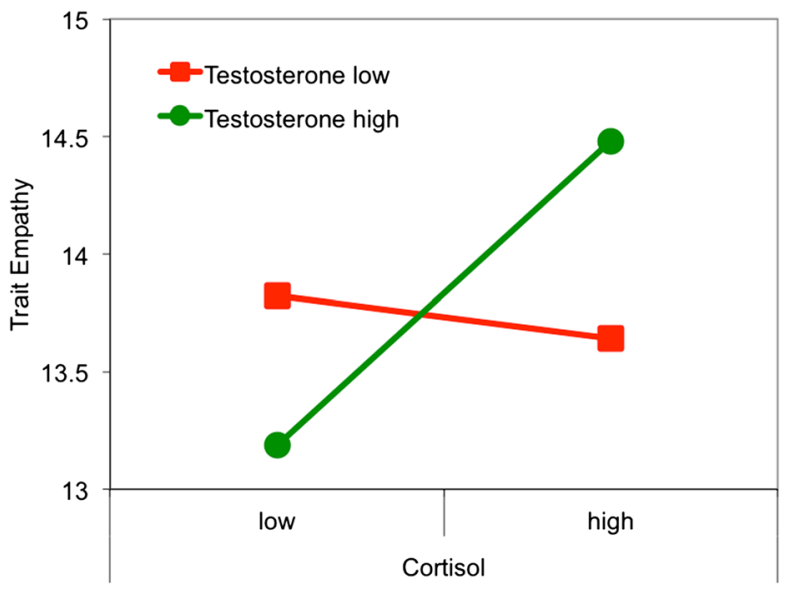

ran a hierarchical linear regression combining the two sexes: empathy was the dependent variable and testosterone (raw data, standardized within sex), cortisol (log-transformed and standardized), sex ( -1 for females and 1 for males) and their interaction were the potential predictors. In our first model, trait empathy was regressed on sex, cortisol and testosterone, which were entered as predictors in Step 1. All two-way interactions were entered in Step 2, while the testosterone by cortisol by sex interaction was inserted in Step 3. Although all three models were statistically significant, only the predictors introduced in Step 2 increased the amount of variance explained in trait empathy $\left[\left(\Delta \mathrm{F}(3,446)=2.613, p=0.051, \Delta \mathrm{R}^{2}=1.7 \%\right]\right.$, while adding the three-way interaction in Step 3 did not increase the variance explained in trait empathy $[(\Delta \mathrm{F}$ $(1,445)=1.848, p=0.175]$. From Step 1 it emerged that women scored higher on empathy $[\beta=-.173, p<.001]$ and cortisol positively predicted empathy $[\beta=.114$, $p=.020]$. In Step 2, the main effect of sex remained significant $[\beta=-.176, p<.001]$, while the main effect of cortisol was qualified by a positive interaction with testosterone $[\beta=.102, p=.045] .{ }^{3}$ When outliers were included the interaction between cortisol and testosterone at Step 2 was not significant $[\beta=-.091, p=.074]$. Simple slope analyses revealed that the slope associated with low cortisol (i.e. 1 SD below the mean) -but not the slope associated with high cortisol $[\beta=.053, p=.483]$ - was statistically different from zero $[\beta=-.143, p=.029]$, indicating that for those individuals with low basal cortisol basal, testosterone negatively predicted trait empathy. In other words, testosterone was associated with empathy only among individuals with low cortisol levels (Fig. 2). We also computed simple slopes between empathy and cortisol one SD below and one SD above the testosterone mean. Similarly to what observed in males only, for baseline testosterone below the mean, the slope did not significantly differ from zero $[\beta=.002, p=.983]$, while a significant effect was found for baseline testosterone one SD above the mean $[\beta=.198, p=.005]$, suggesting that high testosterone levels predicted lower empathy, but only for those individuals with low cortisol levels.

\footnotetext{
${ }^{3}$ Similarly to what observed in the male sample, when running multiple regressions separately for the empathic concern scale and the perspective taking scale, a significant interaction emerged for the former $[\beta=.116, p=.022]$, but not the latter $[\beta=.047, p=.364]$. This suggests that the results were mainly driven by the conjoint effect of testosterone and cortisol on empathic concern.
} 
Fig. 2 Trait empathy as a function of basal testosterone and cortisol levels in men and women. When basal cortisol was low, basal testosterone was negatively related to empathy

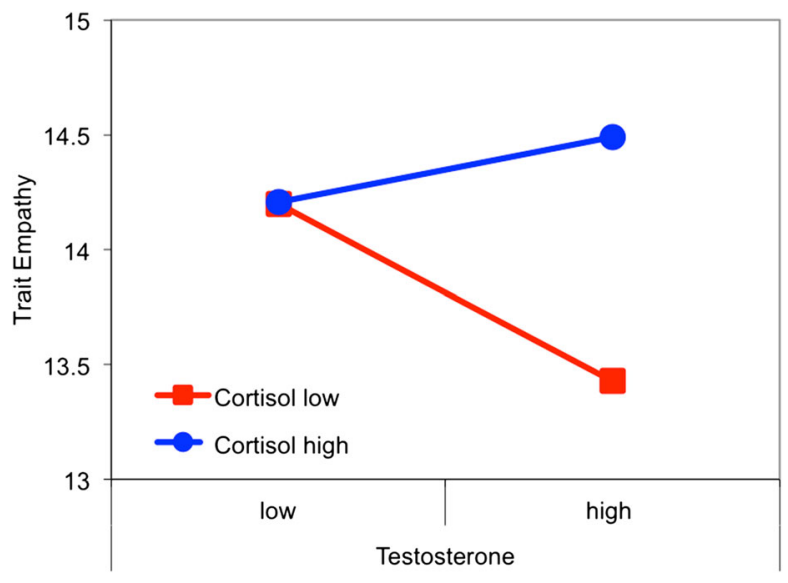

\section{Discussion}

The results of our study support the hypothesized association between basal testosterone, basal cortisol, and empathy, but only with regard to self-reported empathy. In a large adult sample, we found that testosterone was negatively associated with selfreported empathy scores among men and women with low basal cortisol, while this association was not apparent among individuals with high cortisol levels. When baseline testosterone was high, basal concentrations of cortisol were positively related to empathy, so that individuals with high baseline activity in both the HPG and the HPA axis (i.e., high-testosterone and high cortisol) reported higher levels of empathy, whereas individuals with a combination of high testosterone and low cortisol reported lower levels of empathy. This pattern of results, which emerged when data for men and women were analyzed together, remained significant only for men when analyses were run separately for the two sexes. Future studies with a larger number of female participants are needed to better determine the nature of the testosterone-cortisol interaction in predicting trait empathy in women. Further, these hormonal effects might be restricted to affective empathy -and not apply to cognitive empathy- as our results seemed to be driven by one of the two IRI scales, the empathic concern scale. Lastly, the small effect size of our results is not surprising considering our limited simple sampling regime. Future studies involving multiple hormonal (e.g. diurnal cortisol slopes) and behavioral (e.g. repeated empathy measures collected through daily diaries) sampling will be necessary before the practical significance of these findings can be fully understood. Despite these caveats, these results provide the first evidence that the dual hormone hypothesis (Mehta and Josephs 2010) applies to empathy.

A growing body of research has highlighted the relationship between testosterone and empathy. Studies involving the administration of exogenous testosterone in women have provided evidence for a causal relationship between increased testosterone and dampened empathy (Hermans et al. 2006; Van Honk et al. 2011), while correlational studies have reported an inverse relationship between endogenous testosterone levels and various aspects of empathy, such as caring behavior (Baucom et al. 1985), emotional empathy (Harris et al. 1996) and empathic accuracy (Ronay and Carney 2013). 
Reduced basal HPA activity is associated with fearlessness, reduced sensitivity to punishment and threat (Van Honk et al. 2003), and some aspects of aggression (Van Goozen et al. 2007; Shirtcliff et al. 2009; but see, Van Bokhoven et al. 2005), whereas high cortisol levels are associated with anxiety (Brown et al. 1996) and submissive behavior (i.e. social withdrawal and inhibition; Kagan et al. 1988; Goldsmith and Lemery 2000; Klimes-Dougan et al. 2001), but also with higher empathy (reviewed in Shirtcliff et al. 2009).

Our findings are consistent with those of previous studies of testosterone and empathy as well as with those of studies of cortisol and empathy, in particular affective empathy. In our study, high levels of circulating androgens were associated with lower empathic disposition in the absence of any inhibitory action from glucocorticoids, as inferred by the low output of the HPA axis, while they were associated with higher empathic dispositions if paired with high cortisol basal concentrations. Similar effects were observed in earlier studies of the dual hormone hypothesis, wherein a high testosterone/low cortisol profile was predictive of high levels of aggression (Popma et al. 2007), competitiveness (Mehta and Josephs 2010), dominance (Mehta and Josephs 2010), and physiological responses subserving these behaviors (i.e. testosterone reactivity) (Mehta and Josephs 2010; Zilioli and Watson 2012), while a high testosterone/high cortisol profile was predictive of low competitiveness and a sharp decline in testosterone after a social contest (Mehta and Josephs 2010).

Previous studies also showed that dominance-related traits and tendencies typically associated with high testosterone such as status-seeking, reward-seeking, and antisocial tendencies are often associated with lower sensitivity to punishment and with withdrawal, which in turn may reflect an attenuated HPA basal activity (Mehta and Josephs 2010; Terburg et al. 2009). For example, social aggression, a prominent feature of psychopathy, is more common in high testosterone-low cortisol individuals, rather than in all high-testosterone individuals indiscriminately (Terburg et al. 2009). The results of our study suggest that lower empathy in high-testosterone/low-cortisol individuals may be a factor that contributes to the expression of their assertive or aggressive tendencies (Miller and Eisenberg 1988; Jolliffe and Farrington 2004; Jolliffe and Farrington 2007).

The idea that in some circumstances (e.g. in conjunction with high cortisol) testosterone might be associated with enhanced empathy is congruent with recent evidence showing that high exogenous and endogenous androgens may sometimes promote altruistic behavior (van Honk et al. 2012; Diekhof et al. 2014). For example, Diekhof and colleagues reported that in the context of between-group competition, high testosterone men not only favored outgroup hostility but also promoted ingroup prosocial behavior (Diekhof et al. 2014). These novel findings are in line with the idea that men show stronger ingroup-outgroup biases, especially in terms of motivational stances for engaging in intergroup violence and intragroup favoritisms and protection (McDonald et al. 2012). This hypothesis might in turn explain why certain forms of altruistic behavior (e.g., acts of heroism in public safety) are more frequent in men than women and possibly associated with high testosterone levels (Preston 2013). Why this positive relationship between testosterone and empathy was restricted to individuals with a greater HPA output remains to be clarified. However, as suggested by other authors (Shirtcliff et al. 2009), one explanation is that higher cortisol levels would mirror a state of mild arousal and internal distress that aid empathy. 
The results of our study corroborate previous findings on the dual hypothesis, including its extension to psychopathic traits (Terburg et al. 2009). Because lack of empathy and callousness are typical traits among psychopaths, our findings can be interpreted as further empirical evidence for the joint role of testosterone and cortisol in psychopathy. From this perspective, the lack of a significant effect of steroid hormones on RMET, a measure of cognitive empathy, is not surprising, as previous reports showed that psychopaths do not differ from controls in their RMET performance (Richell et al. 2003) and no negative correlation exists between total RMET and selfreported psychopathic traits in non-clinical populations (Sandvik et al. 2014), which, on the contrary, negatively predict IRI scores (Zágon and Jackson 1994). Further, it is possible that the RMET, which was mainly designed for individuals with mild deficits in social cognition (Baron-Cohen et al. 2001) may not be very sensitive to individual variation within the normal range (e.g., see Voracek and Dressler 2006, but see Van Honk et al. $2011^{4}$ ). Future research on the dual hormone hypothesis and empathy should consider using alternative measures of empathy, such as, for example, the Movie for the Assessment of Social Cognition (MASC), a test in which participants have to infer mental states and feelings of four people filmed while having dinner (Dziobek et al. 2006). Future research should also consider other hormonal measures, for example, cortisol reactivity to stress instead of baseline cortisol, as recent evidence indicates that stress cortisol may modulate prosocial behavior (reviewed in Shirtcliff et al. 2009 and Buchanan and Preston 2014).

In conclusion, we hope that the results of our study will stimulate further research on hormonal influences on empathy and that such research will take into consideration the possible joint effect of HPA and HPG axis hormones. Further, studies are also needed to establish whether the findings we obtained with a particular subject population and with relatively limited measures of self-reported empathy can be extrapolated to other subject populations and to other self-reported and experimental measures of empathy.

\section{References}

Adam, E. K., \& Gunnar, M. R. (2001). Relationship functioning and home and work demands predict individual differences in diurnal cortisol patterns in women. Psychoneuroendocrinology, 26(2), 189-208.

Aiken, L. S., \& West, S. G. (1991). Multiple regression: testing and interpreting interactions. Thousand Oaks: Sage Publications, Inc.

Baron-Cohen, S. (2009). Autism: the empathizing-systemizing (E-S) theory. Annals of the New York Academy of Sciences, 1156(1), 68-80.

Baron-Cohen, S., Wheelwright, S., Hill, J., Raste, Y., \& Plumb, I. (2001). The "Reading the Mind in the Eyes" test revised version: a study with normal adults, and adults with Asperger syndrome or high-functioning autism. Journal of Child Psychology and Psychiatry, 42(2), 241-251.

Baron-Cohen, S., Knickmeyer, R. C., \& Belmonte, M. K. (2005). Sex differences in the brain: implications for explaining autism. Science, 310(5749), 819-823.

Barraza, J. A., \& Zak, P. J. (2009). Empathy toward strangers triggers oxytocin release and subsequent generosity. Annals of the New York Academy of Sciences, 1167(1), 182-189.

\footnotetext{
${ }^{4}$ It remains to be determined whether the negative impact of exogenous testosterone -raised to superphysiological levels in women after administration- on cognitive empathy reported by Van Honk et al. (2011) can be compared with the naturally occurring variations in hormones reported in our study.
} 
Baucom, D. H., Besch, P. K., \& Callahan, S. (1985). Relation between testosterone concentration, sex role identity, and personality among females. Journal of Personality and Social Psychology, 48(5), 1218.

Booth, A., Granger, D. A., \& Shirtcliff, E. A. (2008). Gender-and age-related differences in the association between social relationship quality and trait levels of salivary cortisol. Journal of Research on Adolescence, 18(2), 239-260.

Brown, L. L., Tomarken, A. J., Orth, D. N., Loosen, P. T., Kalin, N. H., \& Davidson, R. J. (1996). Individual differences in repressive-defensiveness predict basal salivary cortisol levels. Journal of Personality and Social Psychology, 70(2), 362.

Buchanan, T. W., \& Preston, S. D. (2014). Stress leads to prosocial action in immediate need situations. Frontiers in Behavioral Neuroscience, 8.

Buchanan, T. W., Bagley, S. L., Stansfield, R. B., \& Preston, S. D. (2012). The empathic, physiological resonance of stress. Social Neuroscience, 7(2), 191-201.

Cale, E. M., \& Lilienfeld, S. O. (2002). Sex differences in psychopathy and antisocial personality disorder: a review and integration. Clinical Psychology Review, 22(8), 1179-1207.

Chapman, E., Baron-Cohen, S., Auyeung, B., Knickmeyer, R., Taylor, K., \& Hackett, G. (2006). Fetal testosterone and empathy: evidence from the empathy quotient (EQ) and the "reading the mind in the eyes" test. Social Neuroscience, 1(2), 135-148.

Dabbs, J. M., Jurkovic, G. J., \& Frady, R. L. (1991). Salivary testosterone and cortisol among late adolescent male-offenders. Journal of Abnormal Child Psychology, 19(4), 469-478.

Davis, M. H. (1983). Measuring individual differences in empathy: evidence for a multidimensional approach. Journal of Personality and Social Psychology, 44(1), 113.

Derntl, B., Finkelmeyer, A., Eickhoff, S., Kellermann, T., Falkenberg, D. I., Schneider, F., et al. (2010). Multidimensional assessment of empathic abilities: neural correlates and gender differences. Psychoneuroendocrinology, 35(1), 67-82.

Diekhof, E. K., Wittmer, S., \& Reimers, L. (2014). Does competition really bring out the worst? Testosterone, social distance and inter-male competition shape parochial altruism in human males. PloS One, 9(7), e98977.

Dziobek, I., Fleck, S., Kalbe, E., Rogers, K., Hassenstab, J., Brand, M., et al. (2006). Introducing MASC: a movie for the assessment of social cognition. Journal of Autism and Developmental Disorders, 36(5), 623-636.

Fabes, R. A., Eisenberg, N., \& Eisenbud, L. (1993). Behavioral and physiological correlates of children's reactions to others in distress. Developmental Psychology, 29(4), 655.

Goldsmith, H. H., \& Lemery, K. S. (2000). Linking temperamental fearfulness and anxiety symptoms: a behavior-genetic perspective. Biological Psychiatry, 48(12), 1199-1209.

Gonzalez-Liencres, C., Shamay-Tsoory, S. G., \& Brüne, M. (2013). Towards a neuroscience of empathy: ontogeny, phylogeny, brain mechanisms, context and psychopathology. Neuroscience \& Biobehavioral Reviews, 37(8), 1537-1548.

Harris, J. A., Rushton, J. P., Hampson, E., \& Jackson, D. N. (1996). Salivary testosterone and self-report aggressive and pro-social personality characteristics in men and women. Aggressive Behavior, 22(5), 321331.

Hermans, E. J., Putman, P., \& Van Honk, J. (2006). Testosterone administration reduces empathetic behavior: a facial mimicry study. Psychoneuroendocrinology, 31(7), 859-866.

Jackson, P. L., Meltzoff, A. N., \& Decety, J. (2005). How do we perceive the pain of others? A window into the neural processes involved in empathy. NeuroImage, 24(3), 771-779.

Jolliffe, D., \& Farrington, D. P. (2004). Empathy and offending: a systematic review and meta-analysis. Aggression and Violent Behavior, 9(5), 441-476.

Jolliffe, D., \& Farrington, D. P. (2007). Examining the relationship between low empathy and self-reported offending. Legal and Criminological Psychology, 12(2), 265-286.

Kagan, J., Reznick, J. S., \& Snidman, N. (1988). Biological bases of childhood shyness. Science, 240(4849), 167-171.

Klimes-Dougan, B., Hastings, P. D., Granger, D. A., Usher, B. A., \& Zahn-Waxler, C. (2001). Adrenocortical activity in at-risk and normally developing adolescents: Individual differences in salivary cortisol basal levels, diurnal variation, and responses to social challenges. Development and Psychopathology, 13(03), 695-719.

Lutchmaya, S., Baron-Cohen, S., \& Raggatt, P. (2002). Foetal testosterone and eye contact in 12-month-old human infants. Infant Behavior and Development, 25(3), 327-335.

McDonald, M. M., Navarrete, C. D., \& Van Vugt, M. (2012). Evolution and the psychology of intergroup conflict: the male warrior hypothesis. Philosophical Transactions of the Royal Society, B: Biological Sciences, 367(1589), 670-679. 
Mehta, P. H., \& Josephs, R. A. (2010). Testosterone and cortisol jointly regulate dominance: evidence for a dual-hormone hypothesis. Hormones and Behavior, 58(5), 898-906. doi:10.1016/j.yhbeh.2010.08.020.

Meier, L. L. (2008). IRSE. Interactions in multiple linear regression with SPSS and excel (1.6 ed.).

Miller, P. A., \& Eisenberg, N. (1988). The relation of empathy to aggressive and externalizing/antisocial behavior. Psychological Bulletin, 103(3), 324.

Nakayama, Y., Takahashi, T., Wakabayashi, A., Oono, H., \& Radford, M. H. (2007). Sex differences in the relationship between cortisol levels and the empathy and systemizing quotients in humans. Neuro Endocrinology Letters, 28(4), 445-448.

Popma, A., Vermeiren, R., Geluk, C., Rinne, T., van den Brink, W., Knol, D. L., et al. (2007). Cortisol moderates the relationship between testosterone and aggression in delinquent male adolescents. Biological Psychiatry, 61(3), 405-411. doi:10.1016/j.biopsych.2006.06.006.

Preston, S. D. (2013). The origins of altruism in offspring care.

Preston, S. D., \& De Waal, F. (2002). Empathy: its ultimate and proximate bases. Behavioral and Brain Sciences, 25(01), 1-20.

Richell, R. A., Mitchell, D. G. V., Newman, C., Leonard, A., Baron-Cohen, S., \& Blair, R. J. R. (2003). Theory of mind and psychopathy: can psychopathic individuals read the 'language of the eyes'? Neuropsychologia, 41(5), 523-526.

Ronay, R., \& Carney, D. R. (2013). Testosterone's negative relationship with empathic accuracy and perceived leadership ability. Social Psychological and Personality Science, 4(1), 92-99.

Sandvik, A. M., Hansen, A. L., Johnsen, B. H., \& Laberg, J. C. (2014). Psychopathy and the ability to read the "language of the eyes": Divergence in the psychopathy construct. Scandinavian Journal of Psychology.

Sapienza, P., Zingales, L., \& Maestripieri, D. (2009). Gender differences in financial risk aversion and career choices are affected by testosterone. Proceedings of the National Academy of Sciences, 106(36), 1526815273.

Sapolsky, R. M., Romero, L. M., \& Munck, A. U. (2000). How do glucocorticoids influence stress responses? Integrating permissive, suppressive, stimulatory, and preparative actions. Endocrine Reviews, 21(1), 5589.

Shirtcliff, E. A., Vitacco, M. J., Graf, A. R., Gostisha, A. J., Merz, J. L., \& Zahn-Waxler, C. (2009). Neurobiology of empathy and callousness: implications for the development of antisocial behavior. Behavioral Sciences and the Law, 27(2), 137-171.

Stallings, J., Fleming, A. S., Corter, C., Worthman, C., \& Steiner, M. (2001). The effects of infant cries and odors on sympathy, cortisol, and autonomic responses in new mothers and nonpostpartum women. Parenting, 1(1-2), 71-100.

Tennes, K., \& Kreye, M. (1985). Children's adrenocortical responses to classroom activities and tests in elementary school. Psychosomatic Medicine, 47(5), 451-460.

Terburg, D., Morgan, B., \& van Honk, J. (2009). The testosterone-cortisol ratio: a hormonal marker for proneness to social aggression. International Journal of Law and Psychiatry, 32(4), 216-223. doi:10. 1016/j.ijlp.2009.04.008.

Van Bokhoven, I., Van Goozen, S. H. M., Van Engeland, H., Schaal, B., Arseneault, L., Seguin, J. R., et al. (2005). Salivary cortisol and aggression in a population-based longitudinal study of adolescent males. Journal of Neural Transmission, 112(8), 1083-1096.

Van Goozen, S. H. M., Fairchild, G., Snoek, H., \& Harold, G. T. (2007). The evidence for a neurobiological model of childhood antisocial behavior. Psychological Bulletin, 133(1), 149.

Van Honk, J., Schutter, D. J. L. G., Hermans, E. J., \& Putman, P. (2003). Low cortisol levels and the balance between punishment sensitivity and reward dependency. Neuroreport, 14(15), 1993-1996.

Van Honk, J., \& Schutter, D. J. L. G. (2006). Unmasking feigned sanity: a neurobiological model of emotion processing in primary psychopathy. Cognitive Neuropsychiatry, 11(3), 285-306.

van Honk, J., \& Schutter, D. J. L. G. (2007). Testosterone reduces conscious detection of signals serving social correction implications for antisocial behavior. Psychological Science, 18(8), 663-667.

Van Honk, J., Schutter, D. J., Bos, P. A., Kruijt, A.-W., Lentjes, E. G., \& Baron-Cohen, S. (2011). Testosterone administration impairs cognitive empathy in women depending on second-to-fourth digit ratio. Proceedings of the National Academy of Sciences, 108(8), 3448-3452.

van Honk, J., Montoya, E. R., Bos, P. A., van Vugt, M., \& Terburg, D. (2012). New evidence on testosterone and cooperation. Nature, 485(7399), E4-E5.

Viau, V. (2002). Functional cross-talk between the hypothalamic-pituitary-gonadal and -adrenal axes. Journal of Neuroendocrinology, 14(6), 506-513.

Voracek, M., \& Dressler, S. G. (2006). Lack of correlation between digit ratio (2D: 4D) and Baron-Cohen's "Reading the Mind in the Eyes" test, empathy, systemising, and autism-spectrum quotients in a general population sample. Personality and Individual Differences, 41(8), 1481-1491. 
Yildirim, B. O., \& Derksen, J. J. L. (2012). A review on the relationship between testosterone and the interpersonal/affective facet of psychopathy. Psychiatry Research, 197(3), 181-198.

Zágon, I. K., \& Jackson, H. J. (1994). Construct validity of a psychopathy measure. Personality and Individual Differences, 17(1), 125-135.

Zilioli, S., \& Watson, N. V. (2012). The hidden dimensions of the competition effect: basal cortisol and basal testosterone jointly predict changes in salivary testosterone after social victory in men. Psychoneuroendocrinology, 37, 1855-1865. doi:10.1016/j.psyneuen.2012.03.022. 